\title{
MOLECULAR DOCKING STUDIES ON SCREENING AND ASSESSMENT OF SELECTED BIOFLAVONOIDS AS POTENTIAL INHIBITORS OF COVID-19 MAIN PROTEASE
}

\author{
SHAILENDRA SANJAY SURYAWANSHI ${ }^{1 *}$, POOJA BHAVAKANA JAYANNACHE ${ }^{2}$, RAJKUMAR SANJAY PATIL ${ }^{2}$, \\ PALLED MS $^{1}$, PRIYA SHETTI ${ }^{3}$
}

\author{
1Department of Pharmaceutical Chemistry, 2Department of Pharmacology, 3Department of Pharmacognosy and \\ phytochemistry.KLE College of Pharmacy, Belagavi, KLE Academy of Higher Education and Research, Nehru Nagar, Belagavi, \\ Karnataka, India. Email: shailendrasss80@gmail.com
}

Received: 28 May 2020, Revised and Accepted: 16 July 2020

ABSTRACT

Objectives: The objective of the study was to screen and assess the selected bioactive bioflavonoids in medicinal plants as potential coronaviruses $(\mathrm{CoV})$ main protease (Mpro) inhibitors using molecular docking studies.

Methods: We have investigated several bioflavonoids which include apigenin, galangin, glycitein, luteolin, morin, naringin, resveratrol, and rutin. Nelfinavir and lopinavir were used as standard antiviral drugs for comparison. Mpro was docked with selected compounds using PyRx 0.8 and docking was analyzed by PyRx 0.8 and Biovia Discovery Studio 2019.

Results: The binding energies obtained from the docking of 6LU7 with native ligand, nelfinavir, lopinavir, apigenin, galangin, glycitein, luteolin, morin, naringin, resveratrol, and rutin were found to be $-7.4,-8.3,-8.0,-7.8,-7.3,-7,-7.4,-7.6,-7.8,-6.9$, and $-9 \mathrm{kcal} / \mathrm{mol}$, respectively.

Conclusion: From the binding energy calculations, we can conclude that nelfinavir and lopinavir may represent potential treatment options and apigenin, galangin, glycitein, luteolin, morin, naringin, resveratrol, and rutin found to possess the best inhibitors of CoV disease-19 main protease.

Keywords: Binding energy, 6LU7, Antiviral, Rutin, Coronavirus, Medicinal plants.

(c) 2020 The Authors. Published by Innovare Academic Sciences Pvt Ltd. This is an open access article under the CC BY license (http://creativecommons. org/licenses/by/4. 0/) DOI: http://dx.doi.org/10.22159/ajpcr.2020.v13i9.38485

\section{INTRODUCTION}

The 2019-novel coronavirus (nCoV) is a major source of disaster in the $21^{\text {th }}$ century [1]. New CoV strain was identified in Wuhan, China, in the year 2019. CoVs are an infectious agent and found to cause serious diseases of respiratory tract and digestive tract [2]. The Emergency Committee of the World Health Organization declared an outbreak in China on January 30, 2020, which was considered as Public Health Emergencies of International Concern [3]. Officially, the WHO named this disease as CoV disease (COVID)-2019 on February 11, 2020 [4].

At present, no specific therapies for COVID-19 are available and investigations regarding the treatment of COVID-19 are lacking. Potential combinations of protease inhibitor lopinavir/ritonavir, which is commonly used to treat human immunodeficiency virus, for the treatment of COVID-19-infected patients have been investigated and reported. Furthermore, some other reported antiviral drugs such as remdesivir, umifenovir, tenofovir disoproxil, and lamivudine have been reported for COVID-19 [5].

The outbreaks of COVID-19 highlighted their adaptive potential to the changing environmental conditions and they are classified under "emerging viruses." Knowledge about the structure, metabolic pathways of $\mathrm{CoV}$, and pathophysiology of CoV-associated diseases is important to identify possible drug targets [6-9]. Liu et al. (2020) have successfully crystallized the main protease (Mpro) from COVID-19, which has been structured and repositioned in the Protein Data Bank (PDB) and is accessible by the public. This protease represents a potential target for the inhibition of CoV replication [10].

Flavonoids are the important class of plant secondary metabolites found to possess wide range of biological activities. These natural products were known for their beneficial effects on health long before flavonoids were isolated as the effective compounds [11]. Naturally occurring flavonoids with antiviral activity have been recognized since the 1940s and most of the work related with antiviral compounds revolves around inhibition of various enzymes associated with the life cycle of viruses [12]. Antiviral activity of apigenin, galangin, glycitein, luteolin, morin, naringin, resveratrol, and rutin has been reported in different studies [13-20]. Hence, these bioflavonoids were chosen for the present study.

Literature search revealed that selected bioflavonoids have potent antiviral effect against different viruses and may be effective against COVID-19. Hence, there is a need of screening the selected bioflavonoids against molecular targets of COVID-19 using molecular docking techniques. No studies have been reported on molecular docking studies of selected bioflavonoids against selected target of COVID-19. This promoted us to carry out present research work.

\section{METHODS}

Standard drugs

Nelfinavir and lopinavir were used as standard for comparison.

\section{Bioactive bioflavonoids}

Apigenin, galangin, glycitein, luteolin, morin, naringin, resveratrol, and rutin used as ligands.

\section{Software}

PyRx 0.8, Biovia Discovery Studio 2019, Molsoft, and MarvinSketch.

Determination of drug-likeness properties of selected ligands In our study, we have selected bioflavonoids as ligands. To find out drug-like properties of each ligand, we have followed the Lipinski's rule of five, which states that molecules with poor permeation and 
oral absorption have molecular weights $>500, C \log p>5$, more than 5 hydrogen-bond donors, and more than 10 acceptor groups $[16,17]$. Data of drug likeness profile of selected bioflavonoids were determined with adherence to Lipinski's rule of five. The canonical simplified molecular-input line-entry system was retrieved from PubChem and used in Molsoft software to obtain drug likeness score [21,22]

\section{Preparation of macromolecules}

Structure of COVID-19 3clpro/Mpro (PDB ID: 6LU7) macromolecule was retrieved from PDB (https://www.rcsb.org/), website in pdb format. The retrieved protein is associated with water molecules and hetero atoms. All hetero atoms, water molecules and native ligand were removed using Discovery studio 2019 to avoid docking interference and saved in the PDB format. The 6LU7 protein contains two chains, $\mathrm{A}$ and C. Chain A contains SARS-CoV-2 main protease enzyme; hence, Chain A was used for macromolecule preparation.

\section{Preparation of ligand}

All the three-dimensional structures of the ligand molecules were retrieved from PubChem (https://pubchem.ncbi.nlm.nih.gov/) in structural data format and converted to PDB format using Discovery studio 2019. In the present study, lopinavir and nelfinavir were used as standard drugs, whereas apigenin, galangin, glycitein, luteolin, morin, naringin, resveratrol, and rutin were used as bioflavonoids.

\section{Determination of active sites}

The amino acids in the active site of a protein were determined using the Biovia Discovery Studio 2019. The determination of the amino acids in the active site was used to analyze docking evaluation results [23].

\section{Molecular docking}

PyRx 0.8 was used for molecular docking. After the completion of docking, AutoDock preferences were obtained for both ligand and target in PDBQT format. The docking analysis was performed by Biovia Discovery Studio 2019. The pose for minimum binding energy was selected as best interaction.

\section{RESULTS}

Drug-likeness properties of selected ligands were calculated. Ligands and drug candidate compounds have been previously selected, based on adherence to Lipinski's rule of five. The drug scanning results were calculated and data are presented in Table 1.
The 6LU7 is the Mpro found in COVID-19, which been structured and repositioned in PDB and can be accessed by the public, as of early February 2020. The PDB ID, structure macromolecules native ligand, and amino acids found in the active site pockets of 6LU7 are presented in Table 2.

Molecular docking analysis of selected bioflavonoids, selected drugs, and its two dimensional interaction with different amino acids on targets is presented in Table 3 .

Molecular docking analysis results for several compounds against 6LU7 and its binding energy/Gibbs energy are presented in Table 4. Graph showed molecular docking results between 6LU7 and several drug candidate compounds (the binding energy value $\Delta G$ is shown in minus $\mathrm{kcal} / \mathrm{mol}$ ) and presented in Figs. 1 and 2 (a to $\mathrm{k}$ ) shows the binding between 6 LU7 ligands.

\section{DISCUSSION}

CoVs are the group of viruses which can infect humans and vertebrate animals. CoV infections affect the respiratory, digestive, liver, and central nervous systems of humans and animals [24]. The present study focused on the main proteases in CoVs PDB ID 6LU7 as potential target proteins for COVID-19 treatment. 6LU7 is the

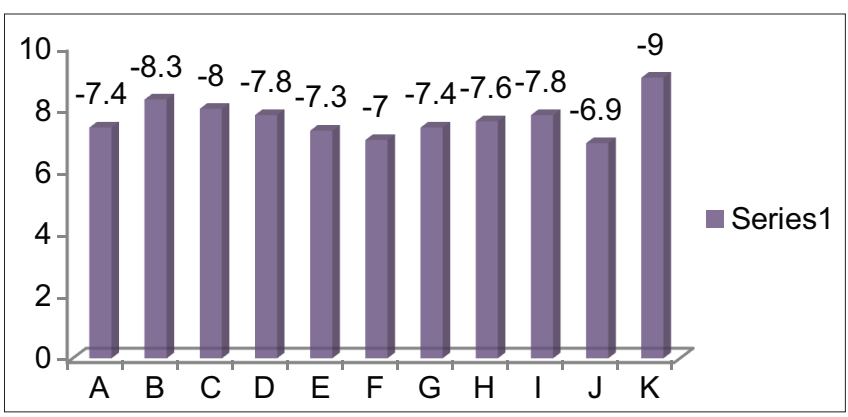

Fig. 1: Graph showing the binding energy value $\Delta \mathrm{G}$ is shown in minus $\mathrm{kcal} / \mathrm{mol}$ on $\mathrm{Y}$-axis versus compounds $\mathrm{A}$ to $\mathrm{K}$ on $\mathrm{X}$-axis. (A - native ligand, B - nelfinavir, C - lopinavir, D - apigenin, E - galangin, F - glycitein, G - luteolin, H - morin, I - naringin, $\mathrm{J}$ - resveratrol, $\mathrm{K}$ - rutin)

Table 1: Drug-likeness properties of selected drugs and ligands

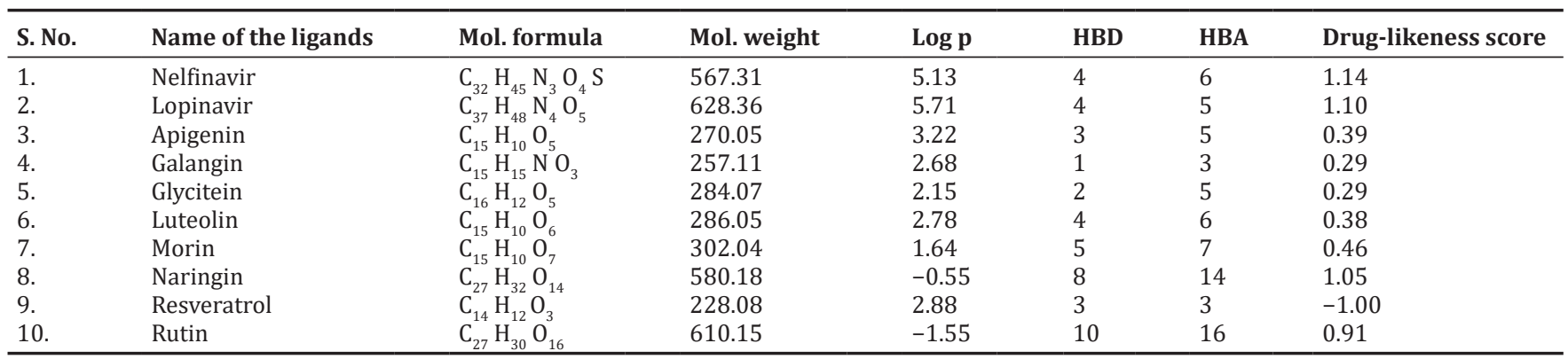

Table 2: PDB ID, target, native ligand, and active sites

\begin{tabular}{llll}
\hline PDB ID & Macromolecule & Native ligand & Active sites \\
\hline 6 LU7 & &
\end{tabular}


Table 3: Molecular docking analysis data of ligands and target

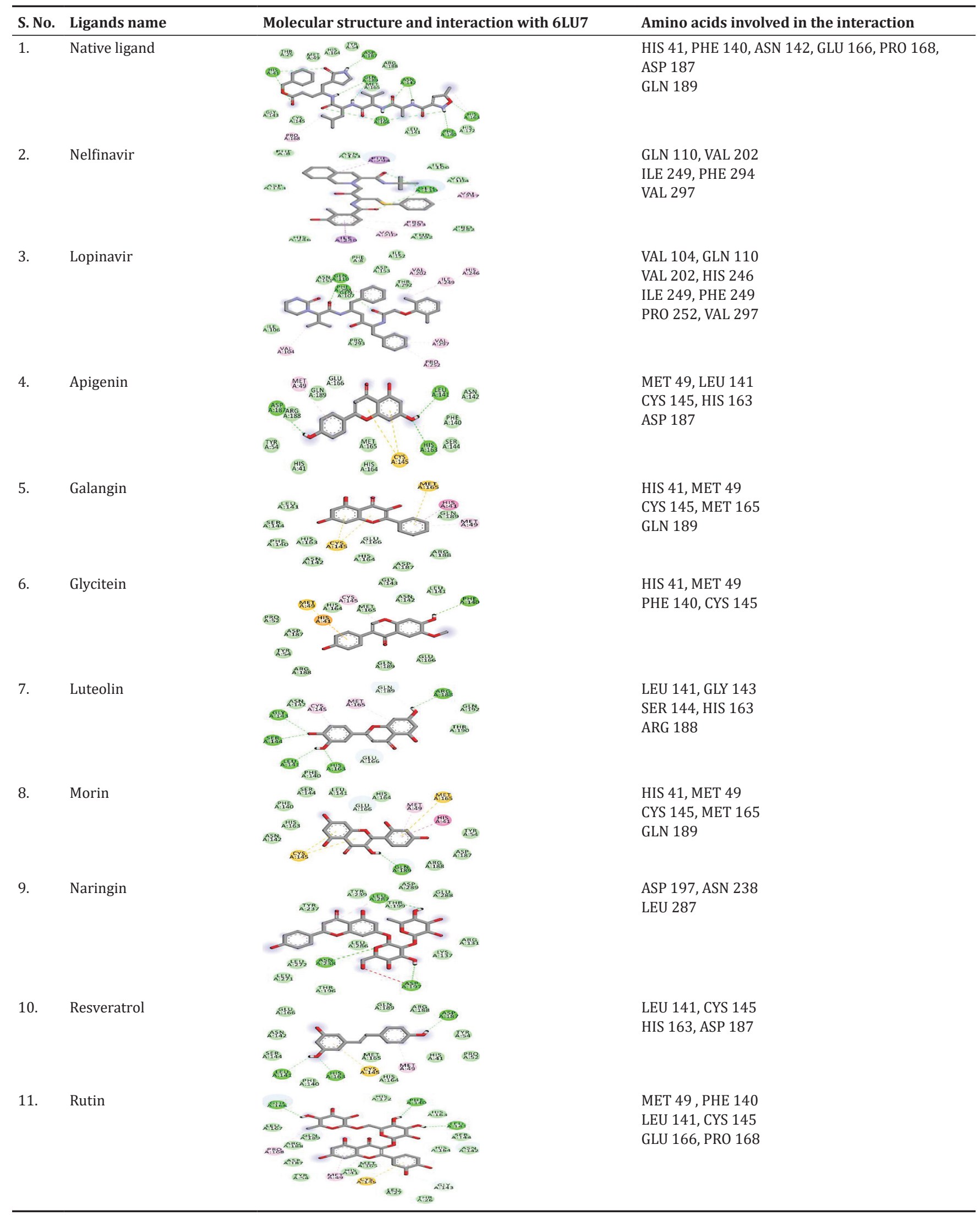

Mpro in COVID-19 that has been structured and repositioned in PDB and has been accessible by the public since early February 2020. The Mpro in CoVs is mainly responsible for proteolytic maturation of the virus and has been examined as a potential target protein to prevent the spread of infection by inhibiting the cleavage of the viral polyprotein [25]. 

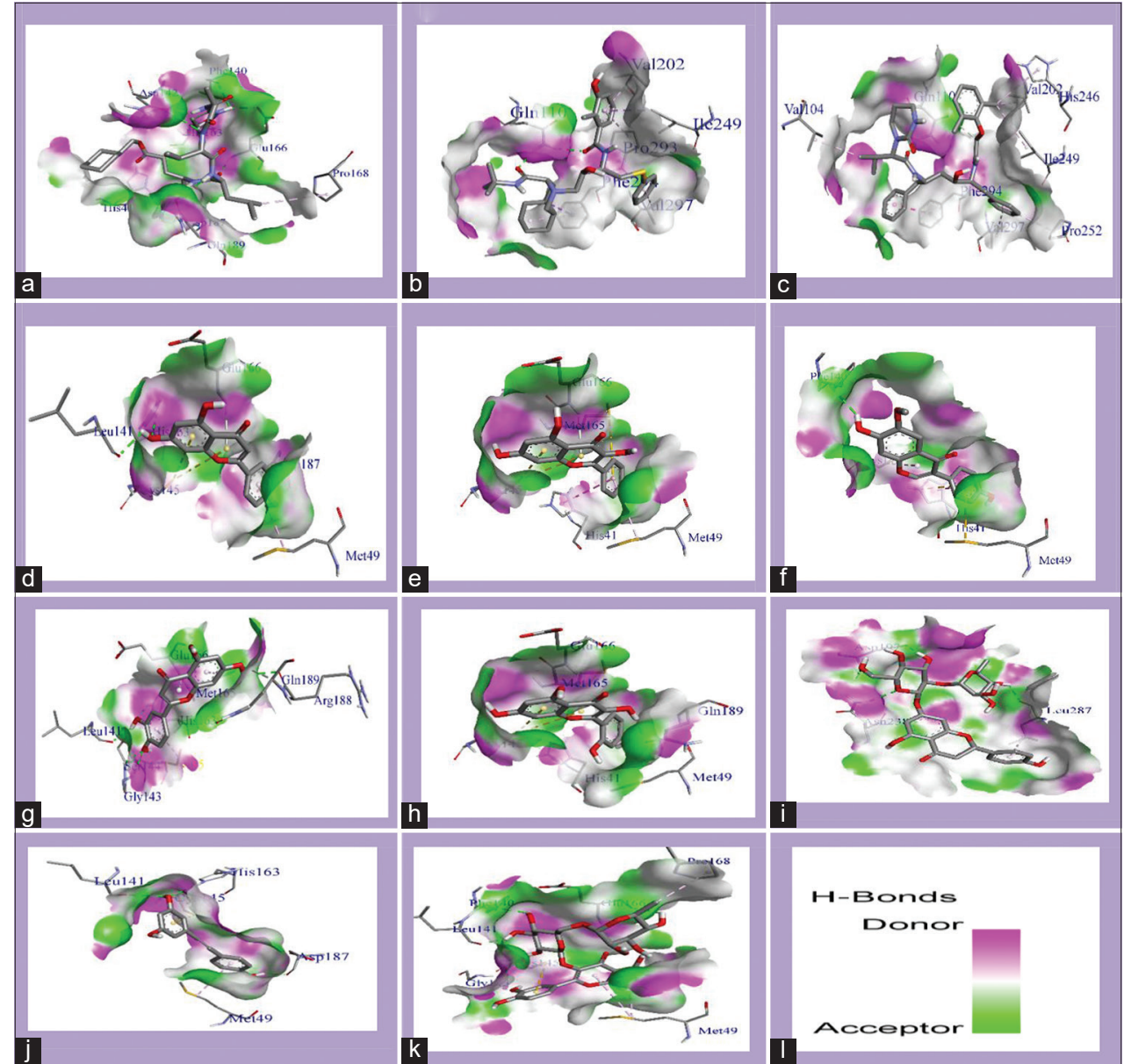

Fig. 2: Docking analysis visualization of 6LU7 binding with (a) native ligand, (b) nelfinavir, (c) lopinavir, (d) apigenin, (e) galangin (f) glycitein, $(g)$ luteolin, $(h)$ morin, $(i)$ naringin, $(j)$ resveratrol, $(k)$ rutin

Table 4: PubChem ID of ligand and binding energy

\begin{tabular}{llll}
\hline Protein & Ligands & PubChem ID & $\begin{array}{l}\text { Binding energy } \\
\text { (kcal/mol) }\end{array}$ \\
\hline 6LU7 & Native ligand & - & -7.4 \\
& Nelfinavir & 92727 & -8.3 \\
& Lopinavir & 64143 & -8.0 \\
& Apigenin & 5280443 & -7.8 \\
Galangin & 5281616 & -7.3 \\
Glycitein & 5317750 & -7 \\
& Luteolin & 5280445 & -7.4 \\
Morin & 5281670 & -7.6 \\
& Naringin & 442428 & -7.8 \\
& Resveratrol & 445154 & -6.9 \\
Rutin & 5280805 & -9 \\
\hline
\end{tabular}

In the present study, we have used nelfinavir and lopinavir as drug standards for comparison. Several bioactive flavonoids, from medicinal plants, have been reported to show antiviral bioactivities [10-12]. We investigated apigenin, galangin, glycitein, luteolin, morin, naringin, resveratrol, and rutin as potential inhibitors of the COVID-19 Mpro. The binding energies obtained from docking 6LU7 with the native ligand, nelfinavir, lopinavir, apigenin, galangin, glycitein, luteolin, morin, naringin, resveratrol, and rutin were $-7.4,-8.3,-8.0,-7.8,--7.3,-7$, $-7.4,-7.6,-7.8,-6.9$, and $-9 \mathrm{kcal} / \mathrm{mol}$, respectively (Table 4 and Fig. 1).

The docking analysis in the present study showed the inhibition potential of several compounds, ranked by affinity $(\Delta G)$; nelfinavir $>$ lopinavir $>$ rutin $>$ apigenin and naringin $>$ morin $>$ luteolin $>$ galangin
$>$ glycitein > resveratrol which were the most recommended flavonoids found in medicinal plants as potential inhibitors of COVID-19 Mpro, which should be explored in future research.

\section{CONCLUSION}

Molecular docking studies of apigenin, galangin, glycitein, luteolin, morin, naringin, resveratrol, and rutin were performed using PyRx 0.8 against main protease enzyme of novel CoV. Binding energies obtained by each molecule were compared with standard antiviral drugs nelfinavir and lopinavir. The study concludes that the binding affinity of rutin is higher, whereas resveratrol has lowest affinity among all the compounds. The nelfinavir and lopinavir may represent potential treatment options, and apigenin, galangin, glycitein, luteolin, morin, naringin, resveratrol, and rutin may be recommended as most potential inhibitors of Mpro of CoV. However, further research is necessary to investigate the effect of selected bioflavonoids on other targets of novel CoVs.

\section{AUTHORS' CONTRIBUTIONS}

The author declares that all the named authors have contributed equally to this article.

\section{CONFLICTS OF INTEREST}

All authors declare that there are no conflicts of interest among the authors.

\section{FUNDING}

This research did not receive any specific grant from funding agencies. 


\section{REFERENCES}

1. Prajapa M, Sarma P, Shekhar N, Avti P, Sinha S, Kaur H, et al. Drug targets for corona virus: A systematic review. Indian J Pharmacol 2020;52:56-65.

2. Malik YS, Sircar S, Bhat S, Sharun K, Dhama K, Dadar M, et al. Emerging novel corona virus (2019-nCoV)-current scenario, evolutionary perspective based on genome analysis and recent developments. Vet Q 2020;40:68-78.

3. Morales AJ, MacGregor K, Kanagarajah S, Patel D, Schlagenhauf P. Going global-travel and the 2019 novel corona virus. Travel Med Infect Dis 2020;33:101578.

4. Zhang D, Wu K, Zhang X, Deng S, Peng B. In silico screening of Chinese herbal medicines with the potential to directly inhibit 2019 novel corona virus. J Integr Med 2020;18:152-8.

5. Lu H. Drug treatment options for the 2019-new corona virus (2019nCoV). Biosci Trends 2020;14:69-71.

6. Yang H, Bartlam M, Rao Z. Drug design targeting the main protease, the Achilles' heel of corona viruses. Curr Pharm Des 2006;12:4573-90.

7. Chang CK, Lo SC, Wang YS, Hou MH. Recent insights into the development of therapeutics against coronavirus diseases by targeting N protein. Drug Discov Today 2016;21:562-72.

8. Saif LJ. Animal coronaviruses: What can they teach us about the severe acute respiratory syndrome? Rev Sci Tech 2004;23:643-60.

9. Alsaadi JE, Jones IM. Membrane binding proteins of corona viruses. Future Virol 2019;14:275-86.

10. Xu Z, Peng C, Shi Y, Zhu Z, Mu K, Wang X. Nelfinavir was predicted to be a potential inhibitor of 2019-nCov main protease by an integrative approach combining homology modelling, molecular docking and binding free energy calculation. BioRxiv 2019;28:1-20.

11. Nijveldt RJ, Nood E, Hoorn D, Boelens PG, Norren KV, Leeuwen P. Flavonoids: A review of probable mechanisms of action apotential applications. Am J Clin Nutr 2001;74:418-25

12. Kumar S, Pandey AK. Chemistry and biological activities of flavonoids: An overview. ScientificWorldJournal 2013;2013:1-6.
13. Ali E, Rahul, Naz F, Jyoti S, Siddique YS. Health functionality of apigenin: A review. Int J Food Propert 2017;20:1197-238.

14. Meyer JJ, Afolayan AJ, Taylor MB, Erasmus D. Antiviral activity of galangin isolated from the aerial parts of Helichrysum aureonitens. J Ethnopharmacol 1997;56:165-9.

15. Zhang L, Chen J, Chai W, Ni M, Sun X, Tian D. Glycitin regulates osteoblasts through TGF- $\beta$ or AKT signaling pathways in bone marrow stem cells. Exp Ther Med 2016;12:3063-7.

16. Qian S, Qian P, Li X. Antiviral activity of luteolin against Japanese encephalitis. Virus 2016;15:112-6.

17. Gravina HD, Tafuri NF, Silva A, Fietto JL, Oliveira TT, Diaz MA et al. In vitro assessment of the antiviral potential of trans-cinnamic acid, quercetin and morin against equid herpesvirus 1. Res Vet Sci 2011;91:58-62.

18. Salehi B, Fokou PV, Sharifi-Rad M, Zucca P, Pezzani R, Martins N et al. The Therapeutic potential of naringenin: A review of clinical trials. Pharmaceuticals 2019;12:1-8.

19. Abba Y, Hasliza H, Hamzah H, Noordin MM. Antiviral activity of resveratrol against human and animal viruses. Adv Virol 2015;2015:1-7.

20. Ganeshpurkar A, Saluja AK. The pharmacological potential of rutin. Saudi Pharm J 2017;25:149-64

21. Lipinski CA, Lombardo F, Dominy BW, Feeney PJ. Experimental and computational approaches to estimate solubility and permeability in drug discovery and development settings. Adv Drug Deliv Rev 2012;64:4-17.

22. Giménez BG, Santos MS, Ferrarini M, Fernandes JP. Evaluation of block buster drugs under the rule-of-five. Pharmazie 2010;65:148-52.

23. Xavier GS, Michael S. Molecular docking studies on antiviral drugs for SARS. IJARCSSE 2015;5:75-9.

24. Xu J, Zhao S, Teng T, Abdalla AE, Zhu W. Systematic comparison of two animal-to-human transmitted human corona viruses: SARS-CoV-2 and SARS-CoV. Viruses 2020;12:244.

25. Liu RX, Zhang B, Jin Z, Yang H. The Crytal Structure of 2019-nCoV Main Protease in Complex with an Inhibitor N3; 2020. Available from: https://www.rcsb.org/structure/6lu7. 